\title{
Prediction Model of Drilling Performance for Percussive Rock Drilling Tool
}

\author{
Dae-Ji Kim, ${ }^{1,2}$ Jaewon Kim, ${ }^{3}$ Booyeong Lee, ${ }^{1}$ Min-Seok Shin, ${ }^{2}$ Joo-Young Oh, ${ }^{2}$ \\ Jung-Woo Cho, ${ }^{2}$ and Changheon Song $\mathbb{B D}^{2}$ \\ ${ }^{1}$ Department of Mechanical Engineering, Hanyang University, 55 Hanyangdaehak-ro, Sangnok-gu, Ansan, Gyeonggi-do 15588, \\ Republic of Korea \\ ${ }^{2}$ Safety System R\&D Group, Korea Institute of Industrial Technology, 15 Jisiksaneop-ro, Hayang-eup, Gyeongsan-si, \\ Gyeongsangbuk-do 38408, Republic of Korea \\ ${ }^{3}$ Mechanical Components and Materials ReD Group, Korea Institute of Industrial Technology, 88-42 Yongju-ro, Jangsu-myeon, \\ Yeongju-si, Gyeongsangbuk-do 36144, Republic of Korea
}

Correspondence should be addressed to Changheon Song; sch8310@kitech.re.kr

Received 2 June 2020; Revised 12 November 2020; Accepted 27 November 2020; Published 12 December 2020

Academic Editor: Patrick W.C. Tang

Copyright (c) 2020 Dae-Ji Kim et al. This is an open access article distributed under the Creative Commons Attribution License, which permits unrestricted use, distribution, and reproduction in any medium, provided the original work is properly cited.

This study suggests a method for quantitatively estimating the drilling performance of the down-the-hole (DTH) hammer during percussive drilling of rock surfaces. A pneumatic dynamic model of the DTH hammer was developed that considers the mass flow rate relations representing the orifice opening areas of the air tube, the piston, and bit flushing channels. A drill bit motion model was developed to represent the dynamics of a drill bit impacted by a dropped piston and explain the impact stress propagation and rock-crushing mechanism. The rock-crushing effect of the drill button bit was measured through a piston drop test. The pneumatic hammer model and drill bit motion model were then combined in a prediction model to determine the impact efficiency according to different rock types (i.e., soft, medium-hard, and very hard). The drilling efficiency was defined as the input value of the prediction model, which was used to simulate the drilling performance of DTH hammers considering the rock type and dynamic effect of the drill bit. Finally, the simulation results were compared with the results of in situ drilling tests for verification.

\section{Introduction}

Percussive drilling tools are used to extract underground resources, such as petroleum, shale gas, and minerals, and shape blasting holes for tunneling and rock excavation. These tools are typically categorized as either down-the-hole (DTH) or top-hammer drilling, depending on the delivery method and location of the impact force. Figure 1 illustrates the mechanisms of the two methods. In top-hammer drilling, the impact force is supplied by a hydraulic drifter. In DTH drilling, the impact force comes from a hammer system operated by compressed air. Top-hammer drilling is mainly used for boring lengths of 1-20 m (e.g., during blasting in mining and civil engineering), although it can be used up to $40 \mathrm{~m}$. Meanwhile, DTH drilling is frequently used for groundwater development and has been recently used for geothermal power generation; it reportedly has a drilling range of up to $4000 \mathrm{~m} \mathrm{[2].}$

Many researchers have focused on improving and developing DTH drilling tools. Song et al. [2] optimized the performance of the drifter used in top-hammer drilling and proposed values of major design factors to maximize the impact power (i.e., a typical performance indicator that is the product of the impact frequency and impact energy). Oh et al. [3] analyzed the hydraulic system to construct a numerical model of a drifter and evaluated the impact frequency and impact energy with respect to the rock properties. Shin and Song [4] performed a pneumatic simulation to analyze a DTH hammer and used the Taguchi method to optimize the percussive drilling performance. 


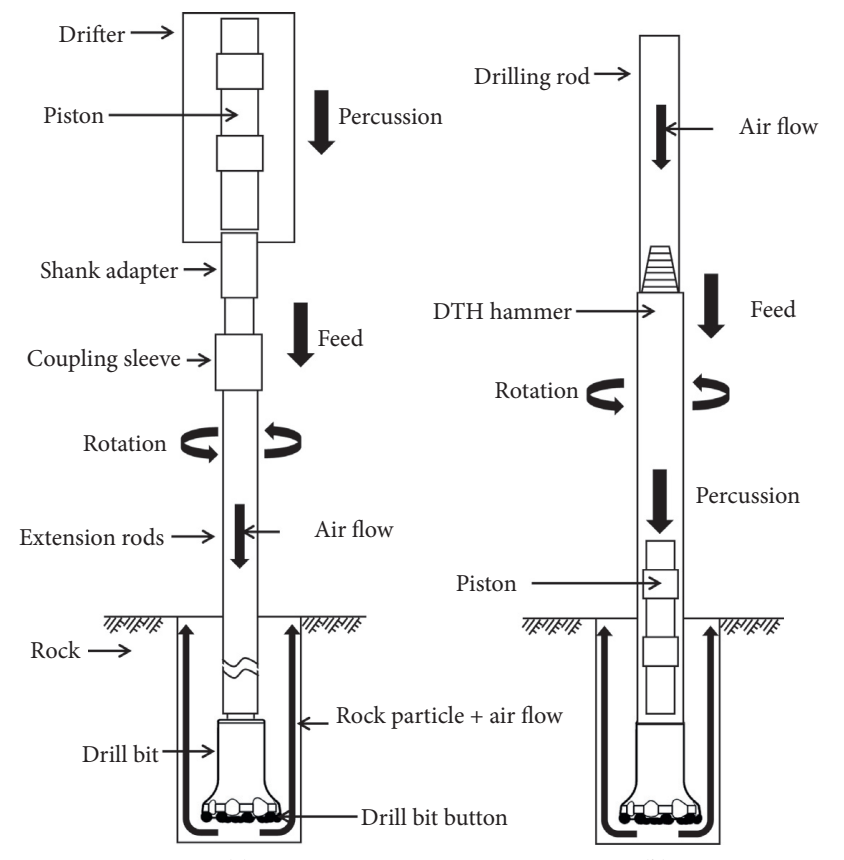

(a)

(b)

FIGURE 1: Drilling mechanisms of two types of percussive drilling systems: (a) top-hammer drilling and (b) down-the-hole drilling [1].

Changgen et al. [5] numerically investigated the changes in rock depth broken by a DTH hammer with respect to the rock type and impact speed. Chiang and Elias [6] used the finite element method (FEM) to simulate the transfer of energy into rock, the interaction between the drill bit and rock, and the rock fragmentation process. Lundberg and Okrouhlik [7] used 1D and 3D FEM to compare the efficiencies of various rock drilling methods, including percussive drilling. Li et al. [8] used the stress wave propagation theory and energy conservation law to analyze the piston reaction force and energy transfer efficiency of the DTH hammer. Li et al. $[8,9]$ considered the dynamics of the drill bit due to piston impact. However, this research was limited because only the damping mode was considered for the system response characteristics owing to the rotational motion of the drill bit and the effect of the rock. Kang et al. [10] proposed a new method for optimizing the arrangement of the drill button bit to enhance the drilling performance.

The above studies mainly focused on evaluating the performance of the DTH hammer and drifter to optimize the design parameters. They did not consider the effects of rock properties (e.g., compressive strength and fractured volume of rock), the drill bit applying the impact force directly on the rock surface, and the interaction between the bit and rock. Although many studies have focused on percussive drilling tools, few have examined the effect of rock properties on the percussive drilling performance of the DTH hammer. In addition, key operating parameters, such as the drilling efficiency, drilling speed, impact frequency, and impact power, have been insufficiently studied. Predicting the drilling performance of a drilling tool requires analyzing the dynamics of the drill bit directly impacting the rock and

ground, as well as of the impact device transmitting the impact force to the drill bit. This analysis must include the dynamic characteristics of the drill bit considering the effect of the rock. However, such analysis is limited by factors including the properties of the rock, the dynamic characteristics of the sensor, the overcapacity due to impact force, and deviation due to impact vibration. Therefore, an analytical model of the drilling platform is required for accurate prediction of the drilling performance considering the characteristics of the drill bit and DTH hammer.

In this study, a new method was developed for predicting the drilling performance of a DTH hammer. A numerical model was developed that combines two previous models: Song et al.'s drill bit-rock interaction model [1] and hammer dynamics model [11]. The proposed model considers the rock fracture effect of the button bit. The performance of the percussive drilling tool is predicted by considering the properties of the percussive system and rock. The nondimensional parameter $\beta$ was defined as the stiffness ratio of the rock and piston and was used to predict the effect of the rock strength on the drilling performance. Therefore, the proposed model can determine the drilling efficiency [1].

The rest of the paper is organized as follows. Section 2 defines the drilling efficiency according to the relationship among the rock, drill bit, and piston during impact, which is used as the input value of the hammer dynamics model. Subsequently, the percussive drilling performance of the DTH hammer was analyzed considering the effect of the rock strength. Section 3 presents the prediction model for the percussive drilling performance, which considers the fracture range of rock types derived from button impact tests. The validity of the model was assessed through comparison with the results of in situ drilling tests.

\section{Combined Simulation Model for Predicting the DTH Hammer Performance}

2.1. Governing Equation for the Impact Hammer Motion. The hammer model uses the following equation of motion for the transfer of pneumatic energy from the piston to the drill bit as impact energy [11], as shown in Figure 2:

$$
m_{p} \ddot{x}_{p}=A_{l}\left(P_{l}-P_{a}\right)-A_{u}\left(P_{u}-P_{a}\right)-m_{p} g \cos \theta+F_{r e}
$$

where $m_{p} \ddot{x}_{p}$ is the impact force on the bit; $A_{l}$ and $A_{u}$ are the pneumatic areas on the lower and upper sides, respectively, of the piston. The atmospheric pressure, lower chamber pressure, upper chamber pressure, piston mass, gravity, and hammer inclination angle are represented by $P_{a}, P_{l}, P_{u}, m_{p}$, $g$, and $\theta$, respectively.

Reaction force $F_{r e}$ due to the stiffnesses of the bit and rock after impact is defined as follows [11]:

$$
F_{r e}=\frac{1}{2} \sqrt{\rho_{p} E_{p}} \cdot A_{l} v_{e},
$$

where $\rho_{p}$ is the density of the piston, $E_{p}$ is the elastic modulus of the piston, and $v_{e}$ is the rebound speed after the bit is impacted. In a percussive drilling system, the piston directly 


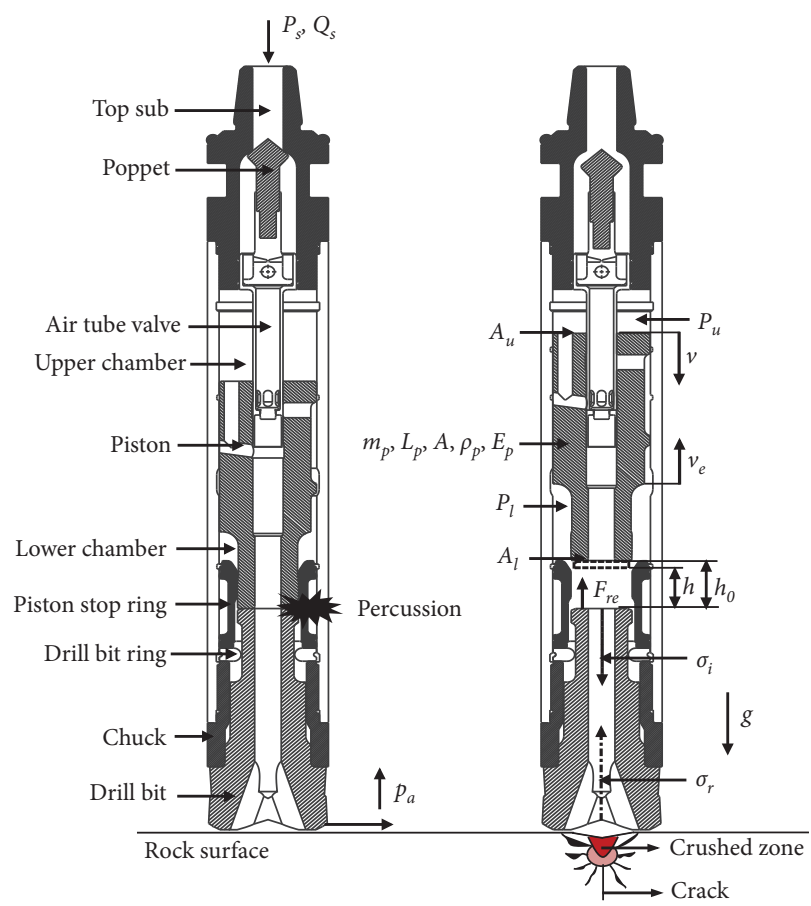

FIgURE 2: Schematic of the rock fracture mechanism by percussive drilling (modified from [1, 11]).

hits the drill bit at speed $v$ (Figure 2). For transmitted impact energy $e_{i n}$, the final descending speed $v$ of the piston during impact with the drill bit can be expressed as follows [1]:

$$
v=\sqrt{\frac{2 e_{i n}}{m_{p}}}
$$

Initial height $h_{0}$ is $v^{2} / 2 g$, and rebound $e_{h}$ can be defined as the ratio between the initial height and postimpact rebound height $h$ of the piston (Figure 2) [1].

$$
e_{h}=\frac{h}{h_{0}}=\frac{v_{e}^{2}}{v^{2}} .
$$

Efficiency $\eta$ of the impact energy transmission is defined as the ratio between the kinetic energy generated from the impact of the piston and the energy transmitted to the rock [1].

$$
\eta=\frac{k u_{m}^{2}}{\rho A_{l} L_{h} v^{2}}=\frac{\left(F_{\max }\right)^{2}}{\rho A_{l} L_{h} v^{2} k}
$$

where $k$ is the rock penetration coefficient index, $u_{m}$ is the maximum penetration depth of the drill bit, $F_{\max }$ is the maximum penetration force, and $L_{h}$ is the drill bit length. Then, $\eta$ can be expressed as follows [1]:

$$
\eta=\frac{2}{\beta} u_{m}^{2}
$$

The dimensionless variable $\beta$ is the stiffness ratio of the rock and piston and indicates the effect of the rock on the motion of the piston transmitting the impact force to the bit [1]. The following modified equation of motion accounts for the movement of the piston that transmits the impact force of the DTH hammer to the bit and $\beta$ (i.e., the effect of the rock).

$$
m_{p} \ddot{x}_{p}=\left\{\begin{array}{l}
A_{l}\left(P_{l}-P_{a}\right)-A_{u}\left(P_{u}-P_{a}\right)-m_{p} g \cos \theta, \quad \text { for } 0<t \leq \tau, \\
A_{l}\left(P_{l}-P_{a}\right)-A_{u}\left(P_{u}-P_{a}\right)-m_{p} g \cos \theta+\frac{1}{2} \sqrt{\rho_{p} E_{p}} \cdot A_{l} v_{e} \sqrt{1-\left(\frac{2}{\beta} u_{m}^{2}\right)}, \quad \text { for } 0<\tau \leq t .
\end{array}\right.
$$

By considering equation (4) and the stiffness of the rock mass after impact, the drilling effect of the piston based on its impact and rebound speeds can be summarized as follows:

$$
m_{p} \ddot{x}_{p}=\left\{\begin{array}{l}
A_{l}\left(P_{l}-P_{a}\right)-A_{u}\left(P_{u}-P_{a}\right)-m_{p} g \cos \theta, \quad \text { for } 0<t \leq \tau, \\
A_{l}\left(P_{l}-P_{a}\right)-A_{u}\left(P_{u}-P_{a}\right)-m_{p} g \cos \theta+\frac{1}{2} \sqrt{\rho_{p} E_{p}} \cdot A_{l} v_{e} \sqrt{1-\left(1-\frac{v_{e}^{2}}{v^{2}}\right)}, \quad \text { for } 0<\tau \leq t .
\end{array}\right.
$$


The duration of the incident stress wave $\tau$ generated by the impact of the piston can be expressed with respect to time $t$ for two conditions, as defined in equation (8): with the transmission of an incident stress wave $(0<t \leq \tau)$ and without $(\tau<t)$. Further, $\tau$ is the duration of the incident stress wave being transmitted to the drill bit via the piston during loading; it is defined relative to time $t$. The loading condition can be defined as $0<t \leq \tau$. If $\tau \leq t$, then the drill bit reaches the maximum penetration depth after the transmission of the incident stress wave to the bit has been completed, and $\tau$ indicates the time when the system switches to the unloading condition.

The falling piston strikes the bit and delivers impact energy $e_{\mathrm{in}}$, whereas $e_{\text {out }}$ is the energy generated when the piston recovers after striking the bit (Figure 3). Thus, the energy due to rock fracture is $e_{\text {in }}-e_{\text {out }}$, and the efficiency can be defined as follows [1]:

$$
\eta=\frac{e_{\text {in }}-e_{\text {out }}}{e_{\text {in }}}
$$

\subsection{Predicted Percussive Drilling Performance according to} Rock Type. The drilling efficiency for the piston and different rock types (i.e., soft rock, medium-hard rock, and hard rock) derived from drilling dynamics [1] were applied to the DTH hammer simulation model to analyze the hammer's performance.

Rock can be classified by its uniaxial compressive strength: <20, 50-120, and >200 MPa for soft, medium-hard, and hard rock, respectively [9]. Figure 3 plots the drilling efficiency estimated from the drilling dynamics with respect to the uniaxial compressive strength of rock (as expressed by $\beta$ ). The results showed that soft rock has low energy (due to fracturing) compared to the energy from the impact of the piston. Thus, soft rock could be drilled with $16 \%$ of the piston energy. The required drilling efficiency increased with the rock strength, and $71 \%$ of the piston energy was needed to crush hard rock. Higher compressive strength for the rock increased the rebound speed of the piston. This implies a loss of impact energy transferred to the rock that would contribute to fracture. In other words, a lower compressive strength of the rock meant that more piston energy contributed to its fracture. This also indicates higher drilling efficiency for soft rock. Thus, higher compressive strength of the rock mass indicates a faster rebound speed for the piston.

Stress propagation theory suggests that, after the impact of the piston, when the incident stress wave (duration $\tau$ ) transforms into a reflected stress wave, the piston rises to the reaction energy $e_{\text {out }}$. This implies that the piston strikes the bit for duration $\tau$ and then rebounds. After the piston rebounds, the displacement of the rock mass reaches its maximum depth owing to the inertia of the bit. The displacement of a striking drill bit was predicted. Figure 4(a) plots the maximum penetration depth for different rock strengths, and Figure 4(b) shows the piston displacement, which is a major performance index for a DTH hammer. The penetration depth of the piston varied with the rock strength (Figure 4(b)). Duration $\tau$ of the incident stress wave

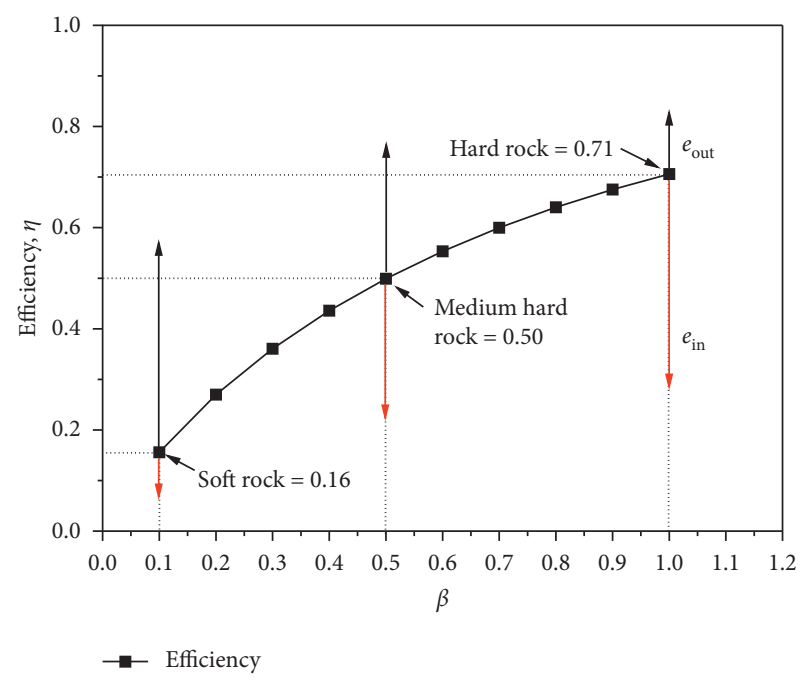

FIgURE 3: Drilling energy transfer efficiency of a DTH hammer with respect to $\beta$.

transmitted by the piston to the bit was short because of the rock strength. A longer $\tau$ reduced the loading time owing to the inertia of the bit, and longer time is needed to reach the maximum penetration depth $u_{m}$ [1].

In other words, as duration $\tau$ increases, greater fracturing of the rock mass can be expected and the drilling efficiency increases. However, despite the increased efficiency, the impact frequency of the percussive drilling tool decreases. This is because the lower strength of the rock increases the drilling depth of the bit and lengthens the rebound time of the piston. This affects the impact stroke, which is the range of motion of the piston of the DTH hammer. A faster rebound shortens the arrival time for the connection to the pneumatic flow path, which is the initial position to prepare the piston for the next stroke.

Table 1 summarizes the impact performance of the DTH hammer for different rock masses. Along with the impact frequency, the table also provides the power required for the DTH hammer to fracture rocks of different strengths.

\section{Prediction Model for the Drilling Performance of the Percussive Drilling System}

The proposed model for predicting the drilling performance of a percussive drilling tool is presented here. The final model is based on the impact performance and buttonstriking tests of a DTH hammer for different rock strengths. A model was developed to predict the impact and drilling performance in conjunction with the drilling dynamics model. The rock-crushing efficacy was considered with and without drill button bits and applied to the DTH hammer dynamics model. Figure 5 shows the calculation procedure of the prediction model. The drilling efficiency of the drill bit is derived from the drilling dynamics model, which considers the effect of the bit-rock interaction. The drilling efficiency is then applied to the hammer dynamics model to evaluate the impact performance according to the dynamic 


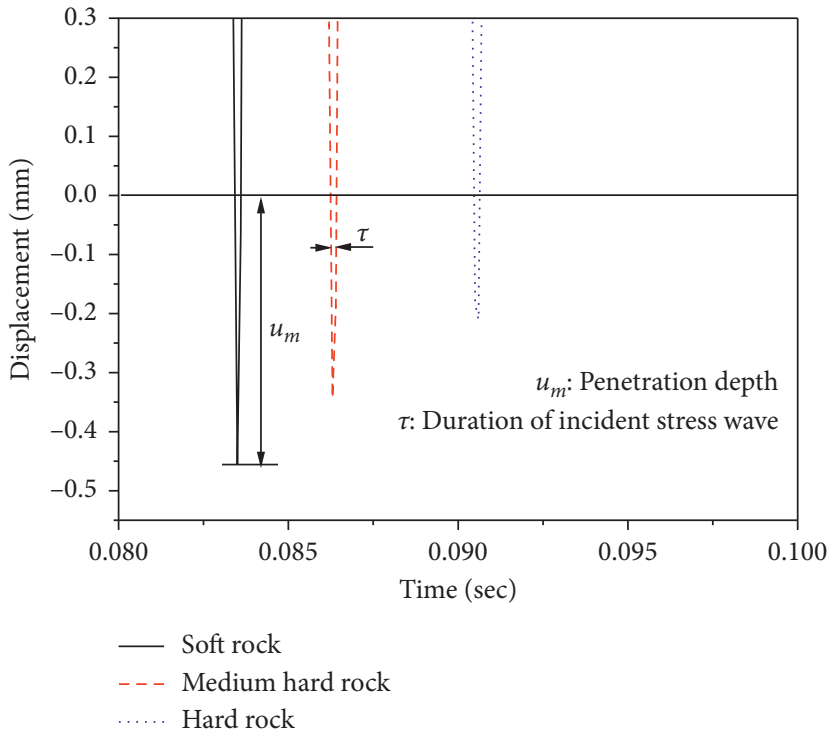

(a)

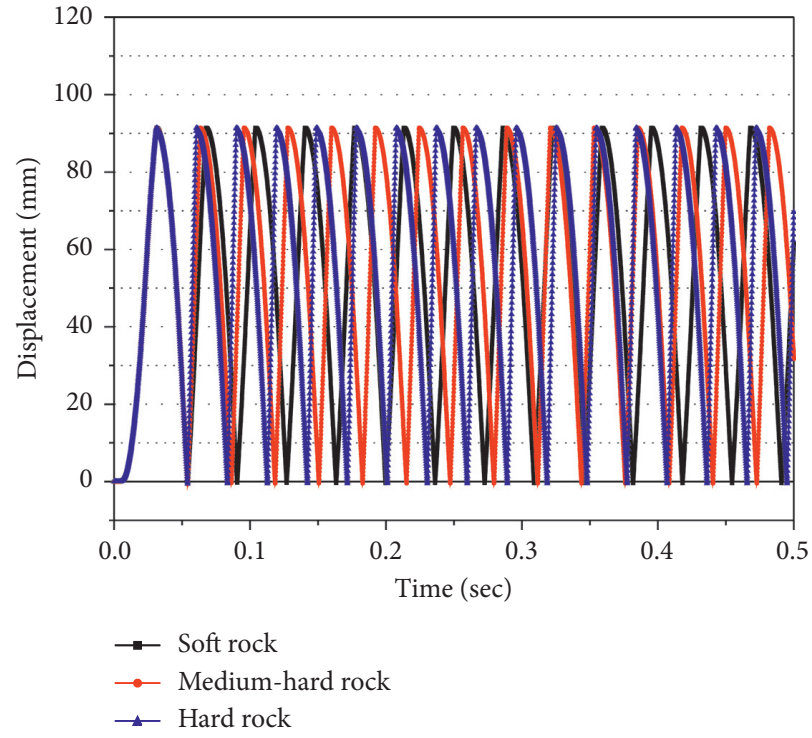

(b)

FIGURE 4: Impact performance of a DTH hammer for different rock types: (a) piston displacement for maximum penetration and (b) piston displacement with time.

TABle 1: Numerical simulation results of a DTH hammer according to rock type.

\begin{tabular}{lcc}
\hline Description & Impact frequency $(\mathrm{Hz})$ & Impact power $(\mathrm{kW})$ \\
\hline Soft rock & 23 & 8.7 \\
Medium-hard rock & 30 & 11.2 \\
Hard rock & 32 & 12.0 \\
\hline
\end{tabular}

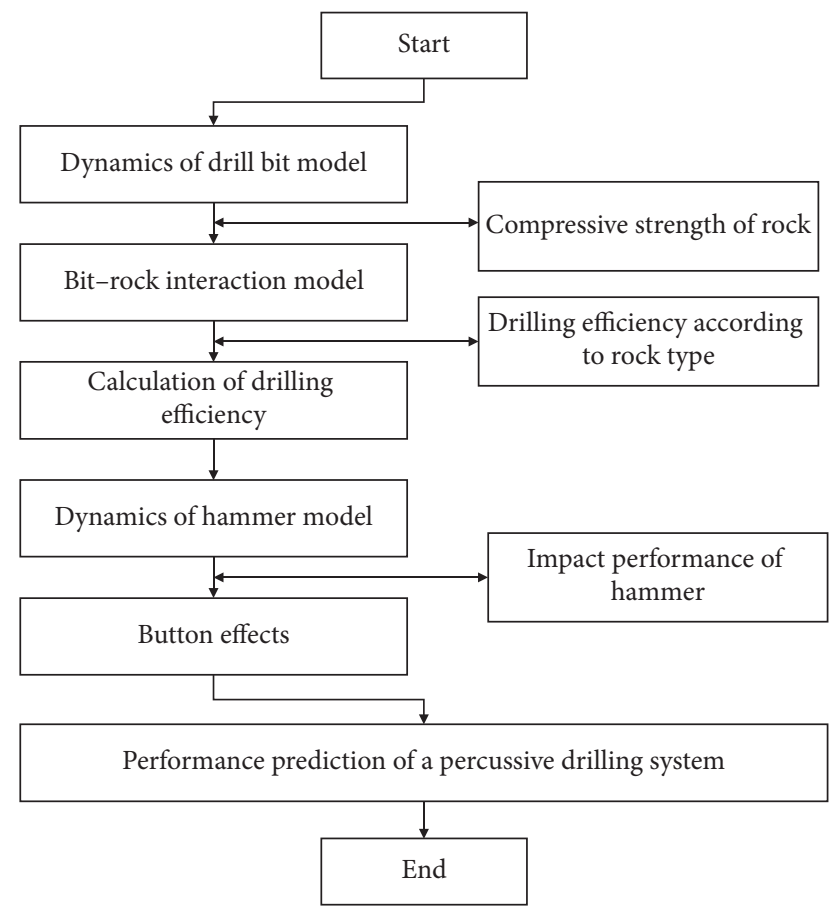

FIGURE 5: Block diagram for predicting the performance of a DTH hammer. characteristics of the drill bit. Finally, the fractured volume of the rock, which is obtained from the button impact test, is applied to the hammer dynamics model to predict the performance of the drilling equipment according to the rock strength.

3.1. Drilling Performance with Buttons. The impact performance of the DTH hammer was analyzed for different rock strengths by correlating the drilling efficiency with the hammer simulation model, which considers the characteristics of the rock mass. The performance was predicted by considering the hammer dynamics through the drilling dynamics model [1] and rock characteristics. During drilling, the reciprocating motion of the piston and bit transmits impact energy that fractures the rock. A button can be inserted under the drill bit to impact the rock surface directly. To predict the performance of the percussive drilling system, an analysis of the performance of the percussive drilling tool (e.g., DTH hammer), location of the drill button bit, and its effect on the subsequent fracturing of the rock is required. As there is no standard for button impact testing, researchers have conducted tests by building vertical or horizontal button test machines [12-16]. In this study, the test system shown in Figure 6 was used for quantitative analysis of the button impact. The system consists of an impact piston to provide the impact force, a drop frame to hold the impact piston, a sliding guide to allow the drop frame to descend, a specimen-fixing cylinder to hold the rock specimen, a hydraulic pressurizing cylinder to apply pressure to the button, and a button that transmits the impact force to the rock mass. Preliminary tests indicated that Hwangdeung granite was suitable as a representative rock sample. It has high compressive strength, so the effect of cracking is small and the experimental error can be reduced. 


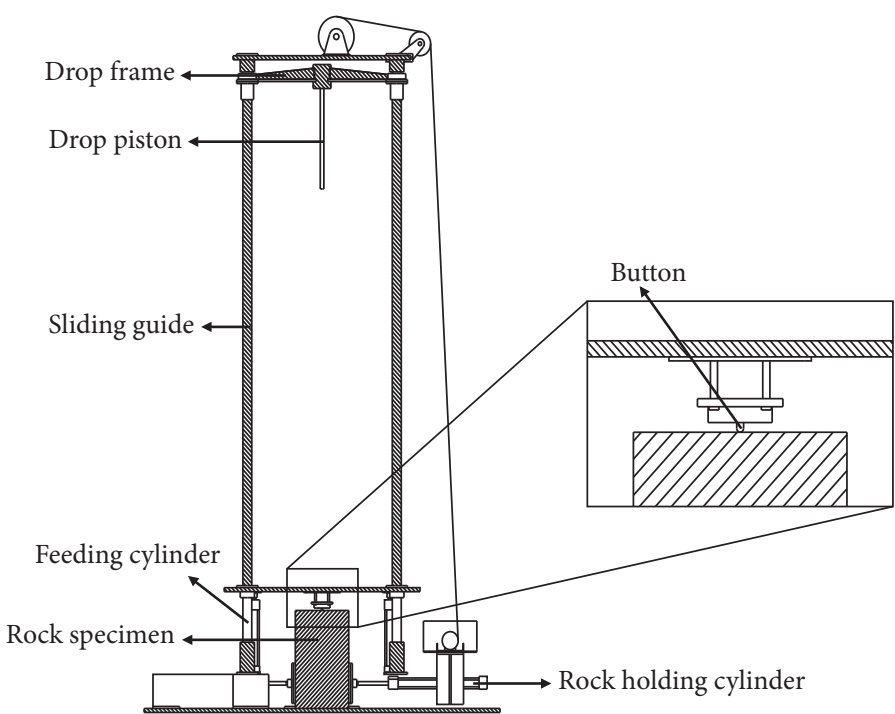

(a)

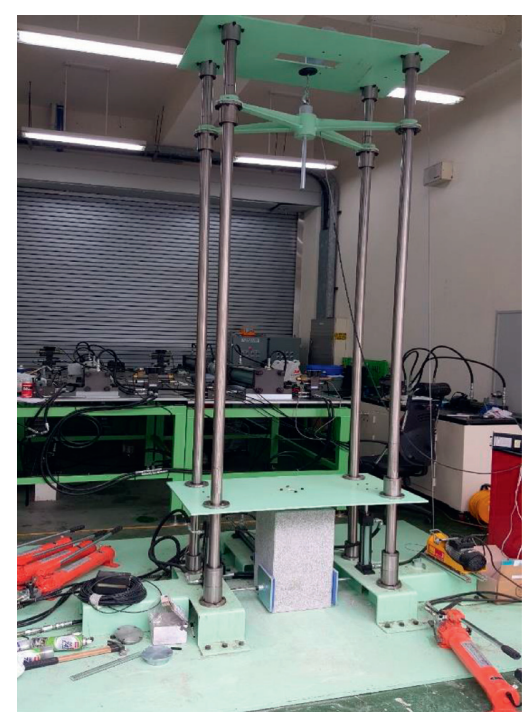

(b)

Figure 6: Schematic of the button impact testing system: (a) cross-section and (b) picture.

Table 2 summarizes the impact test conditions used to verify the effect of the button on fracturing. The feed force for a single button $(2.9 \mathrm{kN})$ was determined by dividing the feed force of the actual vehicle $(43.4 \mathrm{kN})$ by the number of buttons (15). An impact height of $1.5 \mathrm{~m}$ was selected based on the impact energy results obtained from the hammer dynamics. A condition of $\sigma_{c}<\rho_{p} c v_{p}$ was established to calculate the height for crushing a rock mass with a compressive strength of $\sigma_{c}=200 \mathrm{MPa}$. The piston density, longitudinal wave velocity in the piston, and descending speed are represented by $\rho_{p}, c$, and $v_{p}$, respectively.

The falling piston was S45C steel with a density of $7800 \mathrm{~kg} / \mathrm{m}^{3}$ and an elastic modulus of $2.1 \times 10^{11} \mathrm{~N} / \mathrm{m}^{2}$. The previous condition of $\sigma_{c}<\rho_{p} c v_{p}$ was then used to derive the following:

$$
\sigma_{c}<\rho_{p} \sqrt{\frac{E_{p}}{\rho_{p}}} \cdot \sqrt{2 g H},
$$

where the effective height $H$ is

$$
H>\frac{1}{\left(2 g E_{p} \rho_{p}\right)} \sigma_{c}^{2} .
$$

In the present case, equation (11) yields $1.27<H$. Table 3 lists the main properties of the rock used in the impact tests.

The volume of fractures in the rock mass resulting from the button impact test was quantified with a $3 \mathrm{D}$ laser scanner $[12,18]$. The volume was largely irregular; thus, it was quantified by approximation as a spherical cap from the measured crushed volume and area. The volume of the approximated spherical cap can be obtained as follows (Figure 7):

$$
V_{\text {rock }}=\pi \int_{R-h}^{R}\left(R^{2}-y^{2}\right) \mathrm{d} y,
$$

TABle 2: Parameters of the button impact tests.

\begin{tabular}{lc}
\hline Description & Value \\
\hline Button diameter & $18.0 \mathrm{~mm}$ \\
Button height & $22.0 \mathrm{~mm}$ \\
Button material & Tungsten carbide \\
Feed force of actual vehicle & $43.4 \mathrm{kN}$ \\
Feed force of a single & $2.9 \mathrm{kN}$ \\
button & $1.5 \mathrm{~m}$ \\
Drop height & Hwangdeung granite \\
Rock specimen & $300 \mathrm{~mm}(L) \times 300 \mathrm{~mm}(W) \times 600 \mathrm{~mm}$ \\
Rock size & $(H)$ \\
\hline
\end{tabular}

TABLE 3: Mechanical properties of rock used in impact tests.

\begin{tabular}{lc}
\hline Rock specimen & Hwangdeung granite \\
\hline$S_{C}(\mathrm{MPa})$ & 183 \\
$S_{T}(\mathrm{MPa})$ & 9.8 \\
$\rho_{r}\left(\mathrm{~kg} / \mathrm{m}^{3}\right)$ & 2,680 \\
$E_{r}(\mathrm{GPa}) v$ & 42.3 \\
& 0.18 \\
\hline
\end{tabular}

$S_{C}$ : uniaxial compressive strength; $\rho_{r}$ : density; $E_{r}$ : Young's modulus; $v$ : Poisson's ratio [17].

where

$$
R^{2}=r^{2}-(R-h)^{2}
$$

Then, equation (12) can be solved as follows:

$$
V_{\text {rock }}=\pi\left(\frac{1}{2} r^{2} h+\frac{1}{6} h^{3}\right)
$$

The fracture height $h$ of the spherical cap approximated from the fractured volume $V_{\text {rock }}$ is derived as follows: 


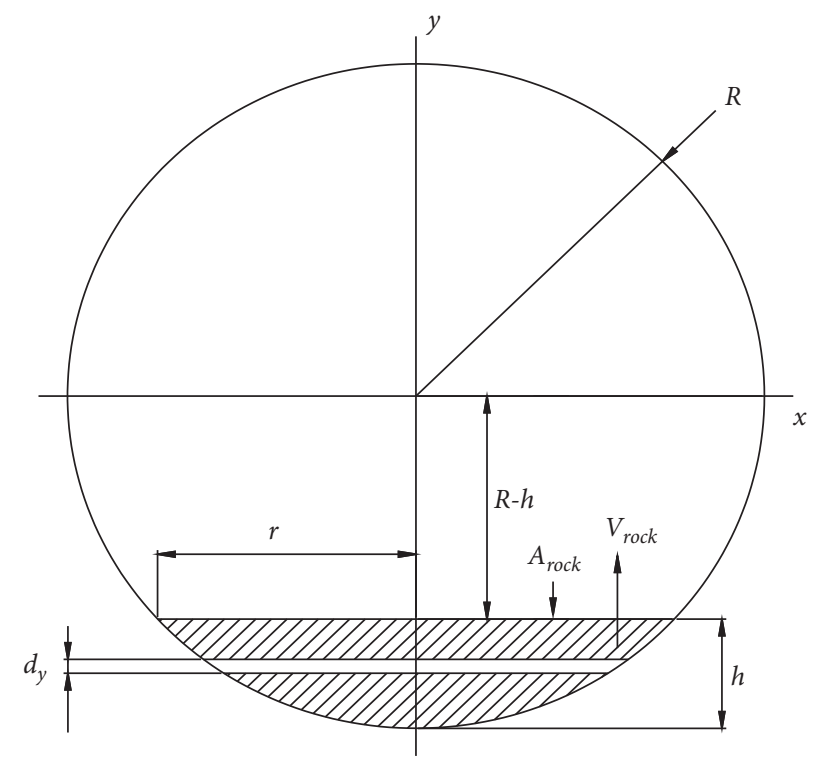

FIGURE 7: Diagram approximating the drilled volume in a rock fractured by a button.

$$
\begin{aligned}
h= & -\frac{1}{3 a}\left(\frac{27 a^{2} d+\sqrt{\left(27 a^{2} d\right)^{2}-4(3 a c)^{3}}}{2}\right)^{1 / 2} \\
& -\frac{1}{3 a}\left(\frac{27 a^{2} d-\sqrt{\left(27 a^{2} d\right)^{2}+4(3 a c)^{3}}}{2}\right)^{1 / 2},
\end{aligned}
$$

where $a=1 / 6, b=0, c=0.5 \times h^{2}$, and $d=-V_{\text {rock }} / \pi$.

Figure $8(\mathrm{a})$ shows the conditions and results of the preimpact test used to confirm the fractured volume overlap of the rock surface. The test showed that the overlap of the fracture area was present if a blow spacing of $1.5 \mathrm{~d}$ was satisfied. However, the overlap of the fracture area did not occur under other conditions. The button impact tests were spaced at twice the button diameter to exclude the effect of connection of the rock fracture volume (Figure $8(\mathrm{~b})$ ). Thus, a test condition of $2.0 \mathrm{~d}$ blow spacing was applied to analyze the rock-crushing effect of the button.

Figure 9 shows the fractured rock surfaces with the blow spacing created during the button impact tests. Table 4 lists the measured fracture volume, depth $h$, and radius $r$ calculated for the button impacts. Percussive drilling relies on a drill bit to make direct contact with the rock. The approximated fracture volume and depth of the drill button bit measured during the impact tests were used to simulate the impact of the button according to $h$ and $r$.

Fifteen tungsten carbide buttons were inserted at the bottom of the drill bit to strike the rock (Figure 10). After a button strikes the rock, the drill bit rotates and hits the rock continuously at constant intervals. The button arrangement in Figure 11(a) caused the impact patterns in Figure 11(b) when the bit was rotated at $60 \mathrm{rpm}$ (resulting in 1560 impacts per minute). The rotation matrix $\mathbf{R}$ of the button according to the revolutions per minute ( $\mathrm{rpm})$ and blows per minute (bpm) is defined as follows:

$$
R=\left[\begin{array}{cc}
\cos \omega t & -\sin \omega t \\
\sin \omega t & \cos \omega t
\end{array}\right]
$$

The impact positions can then be defined as follows:

$$
\left\{\begin{array}{c}
x_{i}^{\prime} \\
y_{i}^{\prime}
\end{array}\right\}=\left[\begin{array}{cc}
\cos \omega t & -\sin \omega t \\
\sin \omega t & \cos \omega t
\end{array}\right]\left\{\begin{array}{l}
x_{i} \\
y_{i}
\end{array}\right\}, \quad i=1,2,3, \cdots, n,
$$

where $x_{i}$ and $y_{i}$ are the coordinates of the reference button, $x^{\prime}{ }_{i}$ and $y_{i}^{\prime}$ are the coordinates of the button after its rotation before the next impact, $\omega$ is the rotation speed, and $t$ is the interval between impacts (Figure 10). Figure 12 shows the result of the complete button fracture model when the coordinates of the button, depth $h$, and fracture radius $r$ are applied.

3.2. Predicted Performance of the Percussive Drilling System via a Simulation Model. The impact performance and drill bit of the DTH hammer were defined and combined with the rock properties to model the drilling performance. The performance can generally be judged according to the drilling speed and drilling volume. The proposed model was used to predict the drilling of a medium-hard rock with a button bit in terms of the drilled volume and depth over time, as depicted in Figure 13. The model predicted a drilling speed of $5.4 \mathrm{~mm} / \mathrm{s}$ under the given conditions.

3.3. Verification of the Simulation Model for Predicting the Drilling Performance. In situ drilling tests were performed to verify the proposed performance prediction model. The DTH hammer and drilling system were optimized [11, 19]. The rock specimens were made of concrete with a compressive strength corresponding to that of medium-hard rock. Compression tests were performed on cylindrical specimens having dimensions of $100 \mathrm{~mm} \times 200 \mathrm{~mm}$ with a 

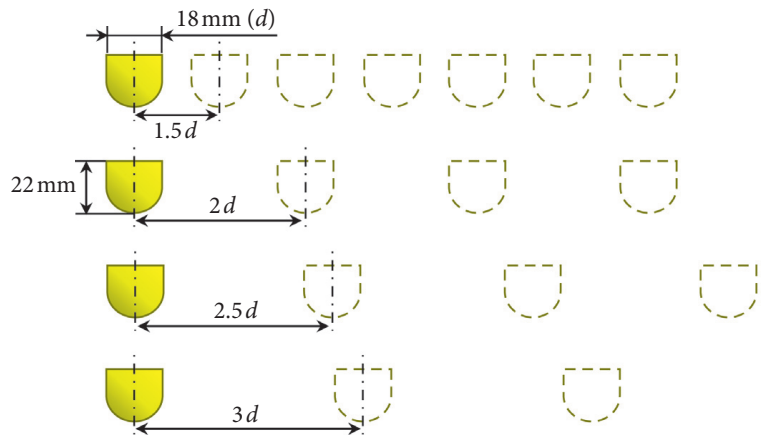

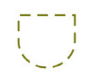

(a)

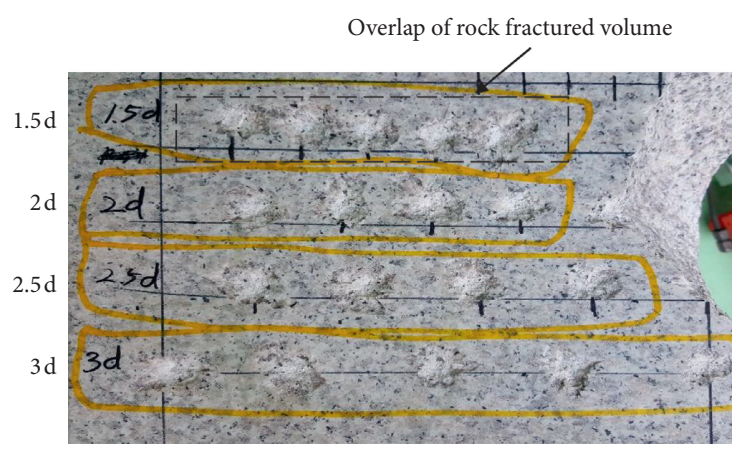

(b)

FIGURE 8: Blow spacing conditions and results of the preimpact test: (a) preimpact test conditions for investigating the effects of rock fracture volume overlap and (b) picture of fractured rock surfaces with blow spacing created during button impact testing.

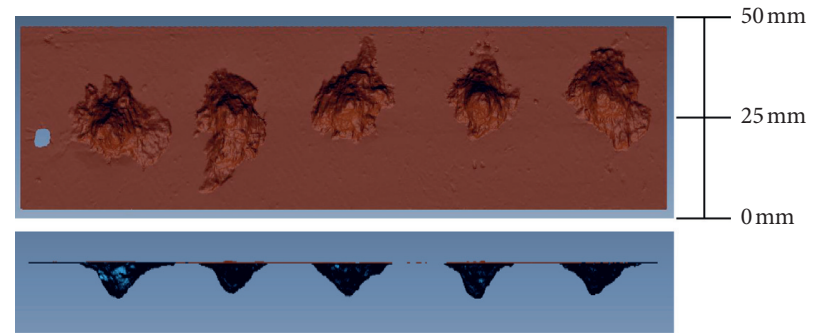

FIGURE 9: Images of rock volumes fractured during button impact testing.

TABLE 4: Results of button impact tests.

\begin{tabular}{lcccc}
\hline Test no. & $V_{\text {rock }}\left(\mathrm{mm}^{3}\right)$ & $A_{\text {rock }}\left(\mathrm{mm}^{2}\right)$ & $r(\mathrm{~mm})$ & $h(\mathrm{~mm})$ \\
\hline 1 & 524.6 & 380.3 & 11.0 & 10.7 \\
2 & 503.5 & 357.0 & 10.1 & 2.7 \\
3 & 503.8 & 317.4 & 8.7 & 3.1 \\
4 & 391.2 & 236.4 & 10.6 & 3.2 \\
5 & 528.7 & 354.2 & 10.2 & 2.9 \\
Average & 490.4 & 329.1 & & 2.9 \\
\hline
\end{tabular}

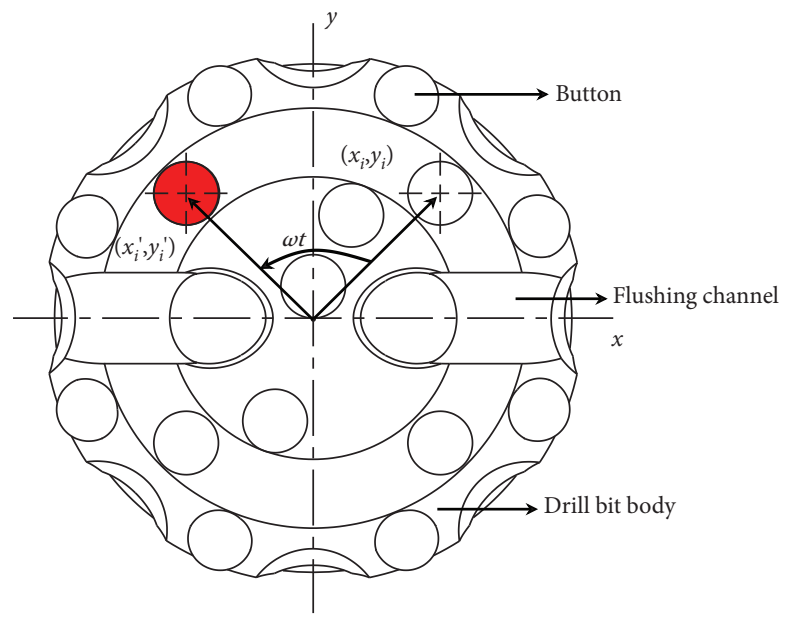

FIGURE 10: Button position with rotation speed $(\omega)$ and impact time $(t)$. 


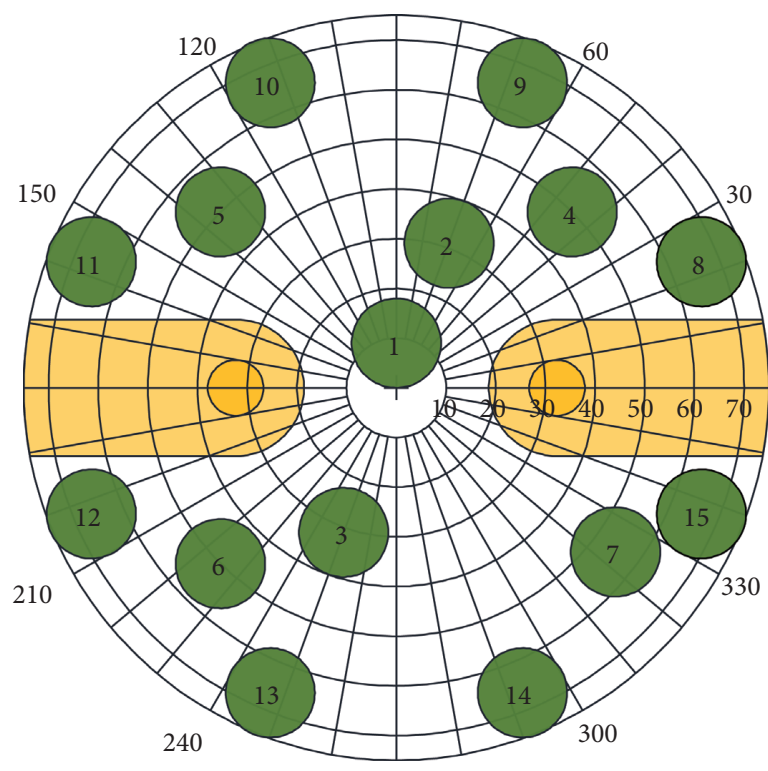

(a)

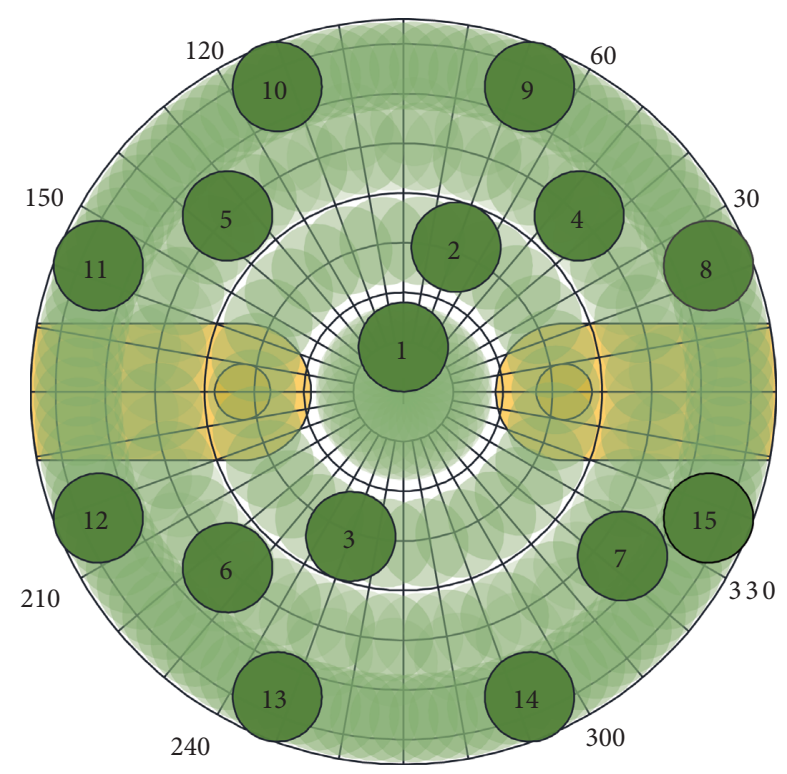

(b)

FIGURE 11: Impact points of buttons depending on the operating conditions: (a) button arrangement and (b) effect during drilling.

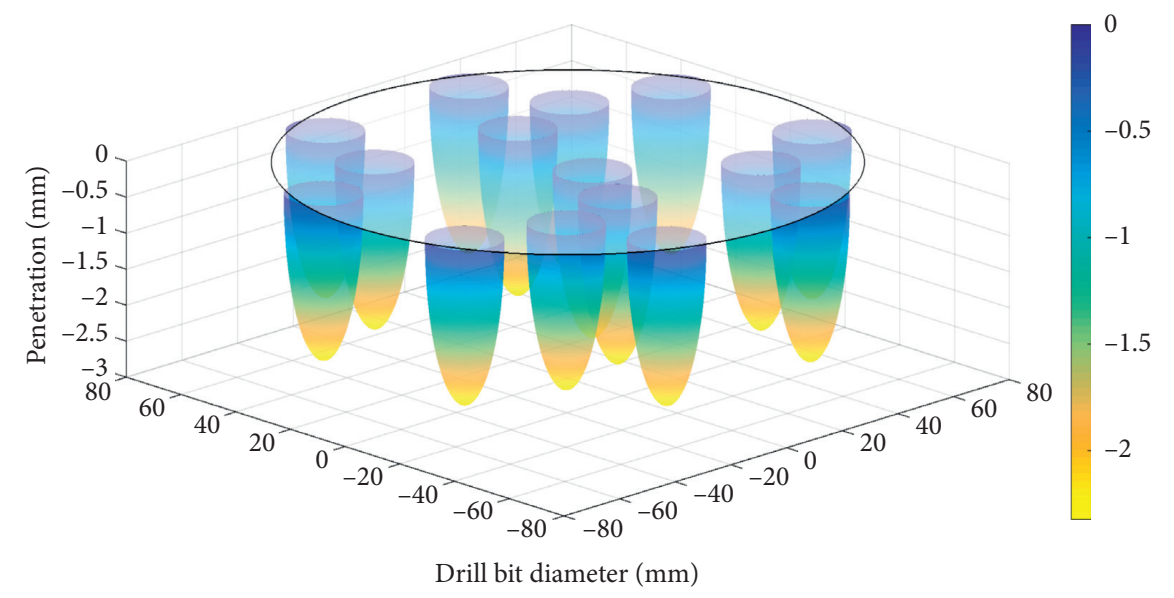

FIgURE 12: Drilled depth (h) and radius (r) of fractures in rock drilled with a button bit.

universal testing machine (Figure 14). The average uniaxial compressive strength was $112.5 \mathrm{MPa}$ (Table 5). Figure 15(a) shows the concrete mass, which had dimensions of $1000 \mathrm{~mm}(L) \times 1,000 \mathrm{~mm}(W) \times 3000 \mathrm{~mm}(H)$. Figure 15(b) shows the DTH drilling system used for the in situ drilling test, and Table 6 lists the operating conditions.

Figure 16(a) shows the rock specimens after DTH drilling, and Figure 16(b) shows the drilling displacement. Section A was used to determine the position of the drilling machine, and section $B$ was used to plot the actual drilling operation. A wire-type displacement meter was used to measure the drilling depth and drilling speed. The impact force of the DTH hammer and vibrations generated by the vehicle during operation caused some difficulties with obtaining drilling data over a long time. Further research is needed to obtain such data, which lay outside the scope of the equipment used in this test.

Figure 17 shows that the measured drilling speed $(5.7 \mathrm{~m} /$ s) and predicted drilling speed (5.4) are similar, which validates the proposed prediction model. The difference between the two values was attributed to the rock fracture effects of the feed force and rotation force fluctuation of the vehicle, in addition to the use of multiple buttons rather than a single button. This model currently reflects only the fracturing of medium-hard rock; further work is warranted to quantify the fracture depths in impact tests on soft and hard rock. In this section, concrete specimens were used for verification. This is because actual rocks have varying mechanical properties due to anisotropy and irregularity [20]. The uniformity and reproducibility of the test were ensured by minimizing the variation in the strength of the specimen. 


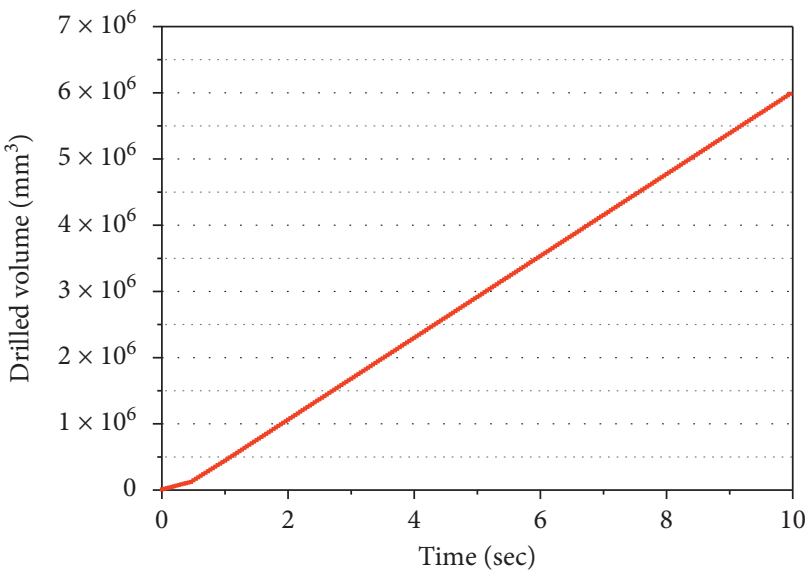

-.- Drilled volume

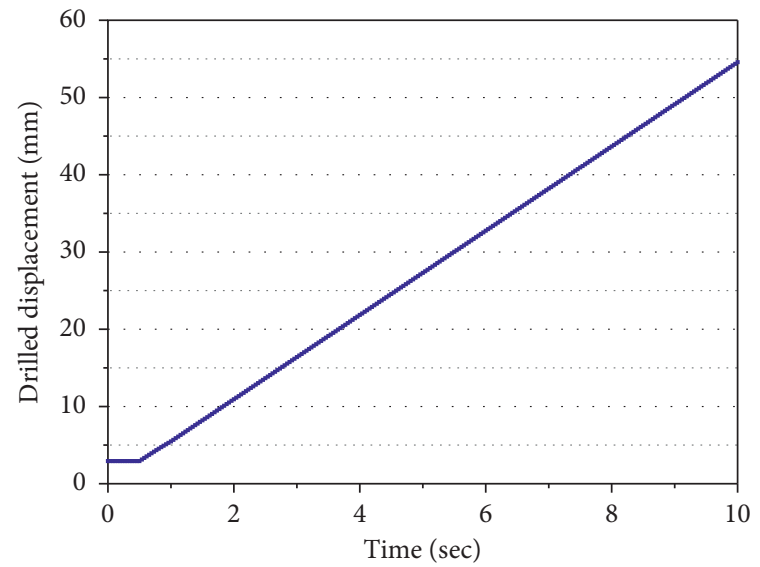

- - Drilled displacement

(a)

(b)

FIGURE 13: Predicted performance of drilling progression considering the effects of a button bit: (a) drilled volume and (b) drilled displacement.

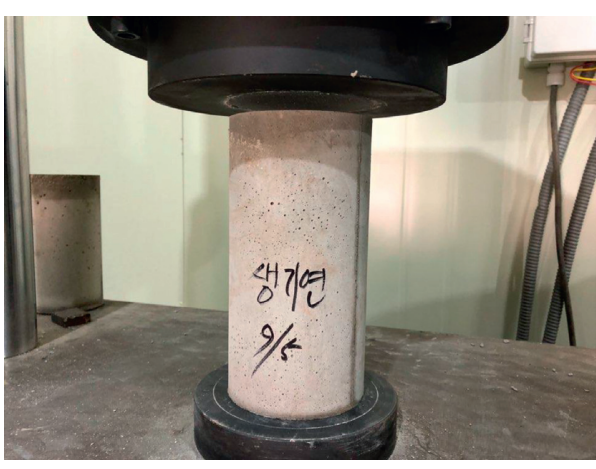

(a)

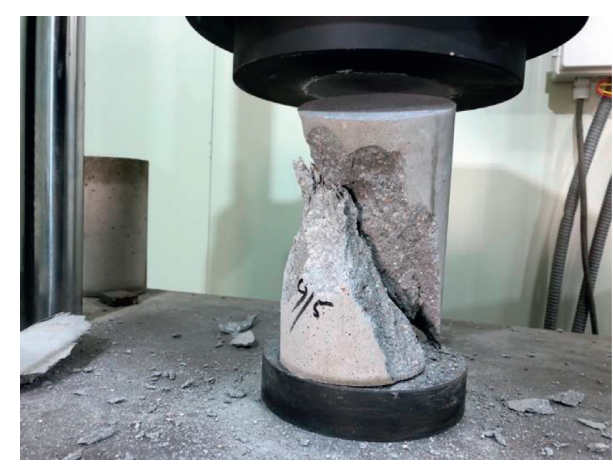

(b)

Figure 14: Uniaxial compression testing of a concrete specimen: (a) test setup and (b) test result.

TABLE 5: Uniaxial compressive strength of concrete specimens.

\begin{tabular}{lr}
\hline Test no. & Value (MPa) \\
\hline 1 & 117.7 \\
2 & 109.9 \\
3 & 115.8 \\
Average & 112.5 \\
\hline
\end{tabular}

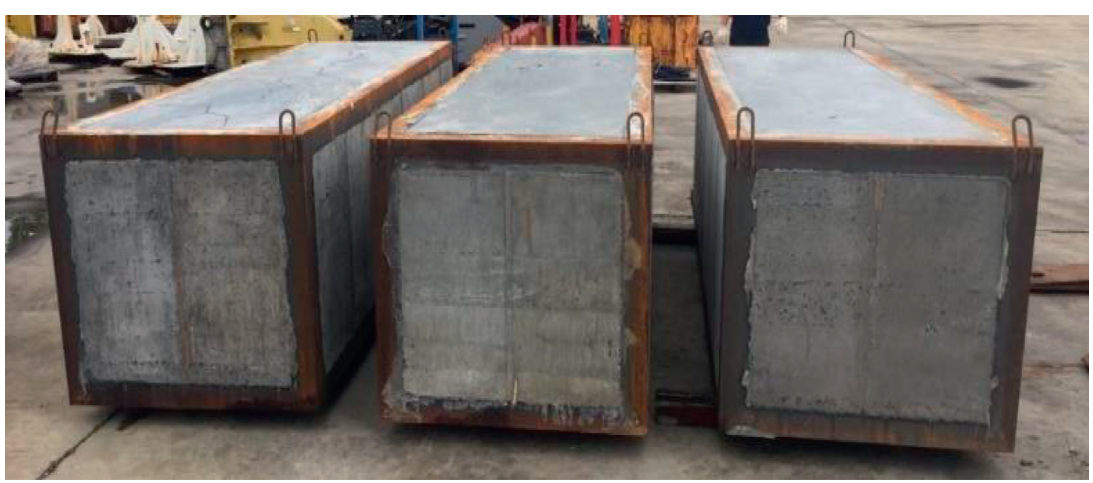

(a)

Figure 15: Continued. 


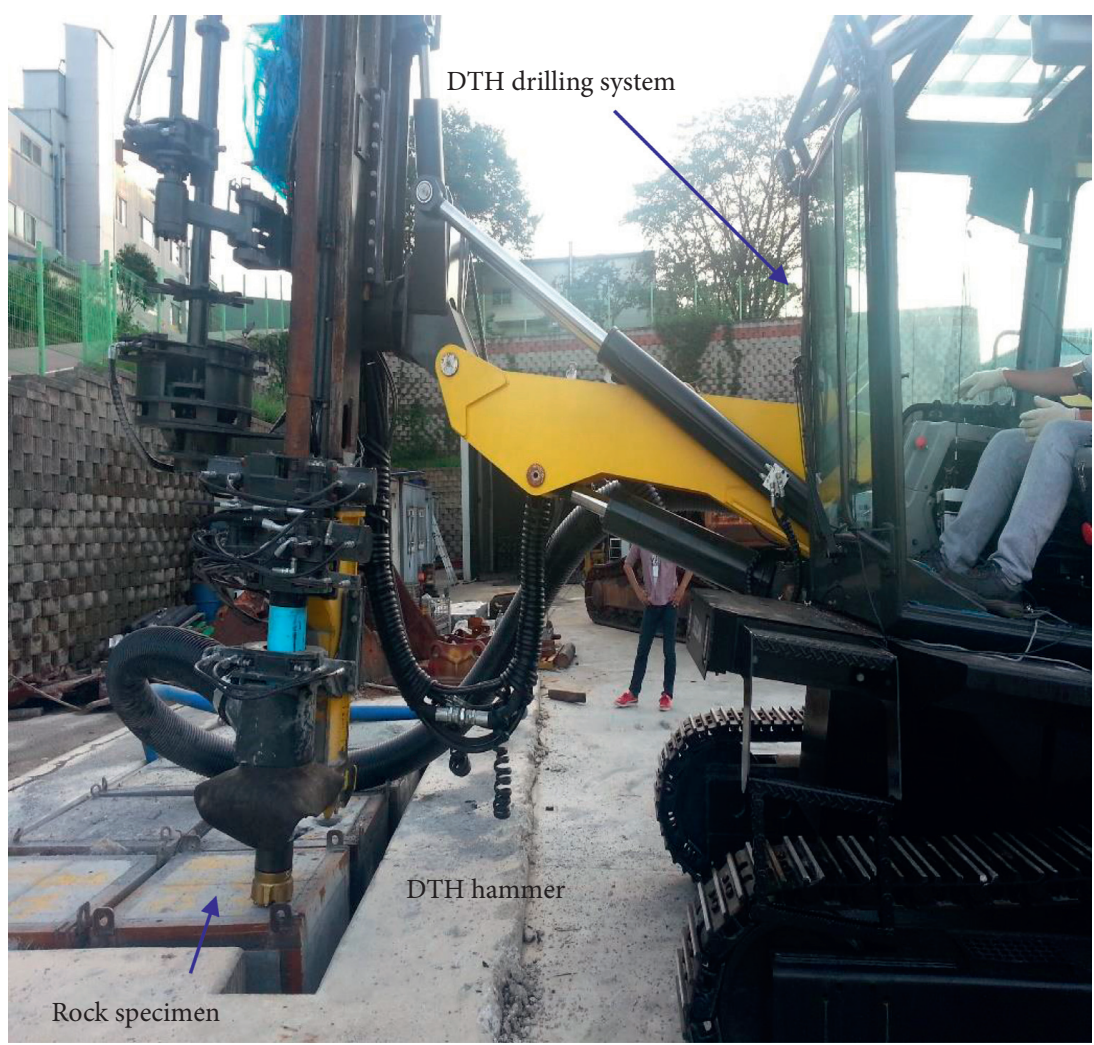

(b)

FIGURE 15: In situ drilling tests: (a) concrete mass to be drilled and (b) percussive rock drilling system.

TABLE 6: Operating conditions of the DTH drilling system.

\begin{tabular}{lr}
\hline Description & Value \\
\hline Feed force & $43.4 \mathrm{kN}$ \\
Rotating pressure & 50.0 bar \\
Compressor pressure & $30.0 \mathrm{bar}$ \\
Compressor flow rate & $35.5 \mathrm{~m}^{3} / \mathrm{min}^{-}$ \\
\hline
\end{tabular}

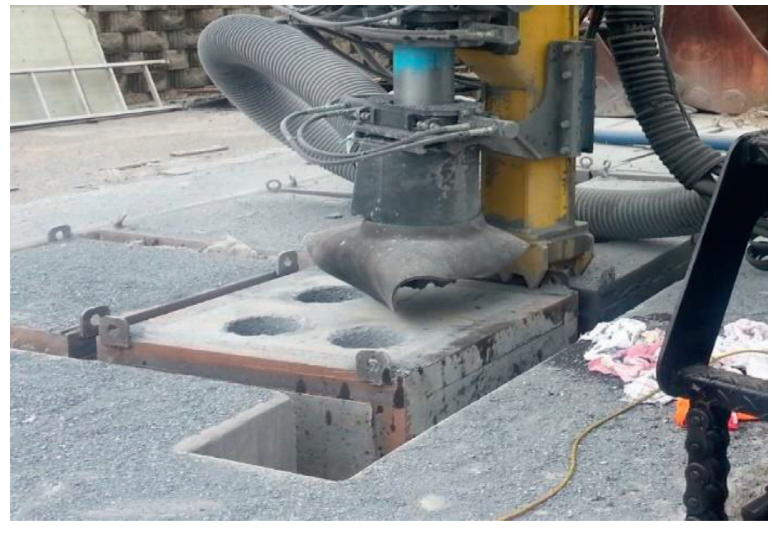

(a)

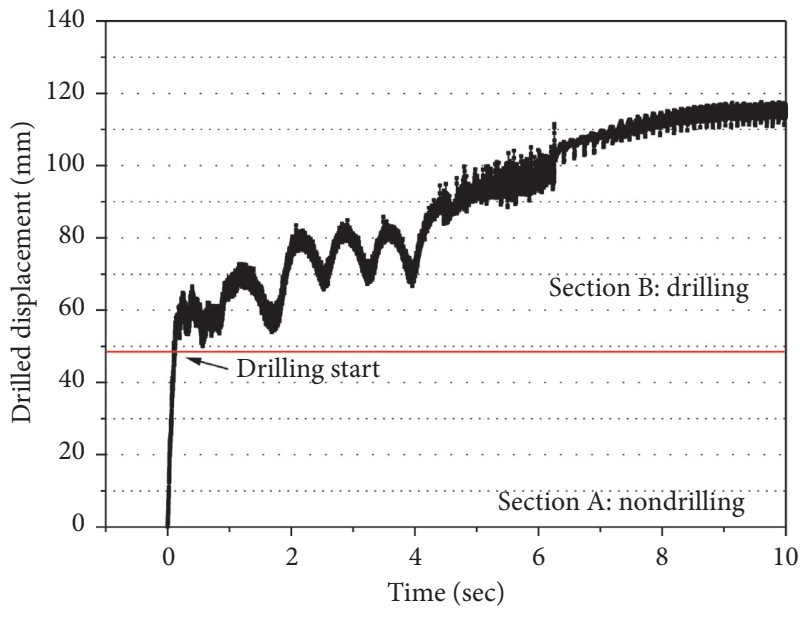

- - D Drilled displacement

(b)

FIGURE 16: Results of the in situ drilling test: (a) concrete mass being drilled and (b) the measured drilled displacement. 


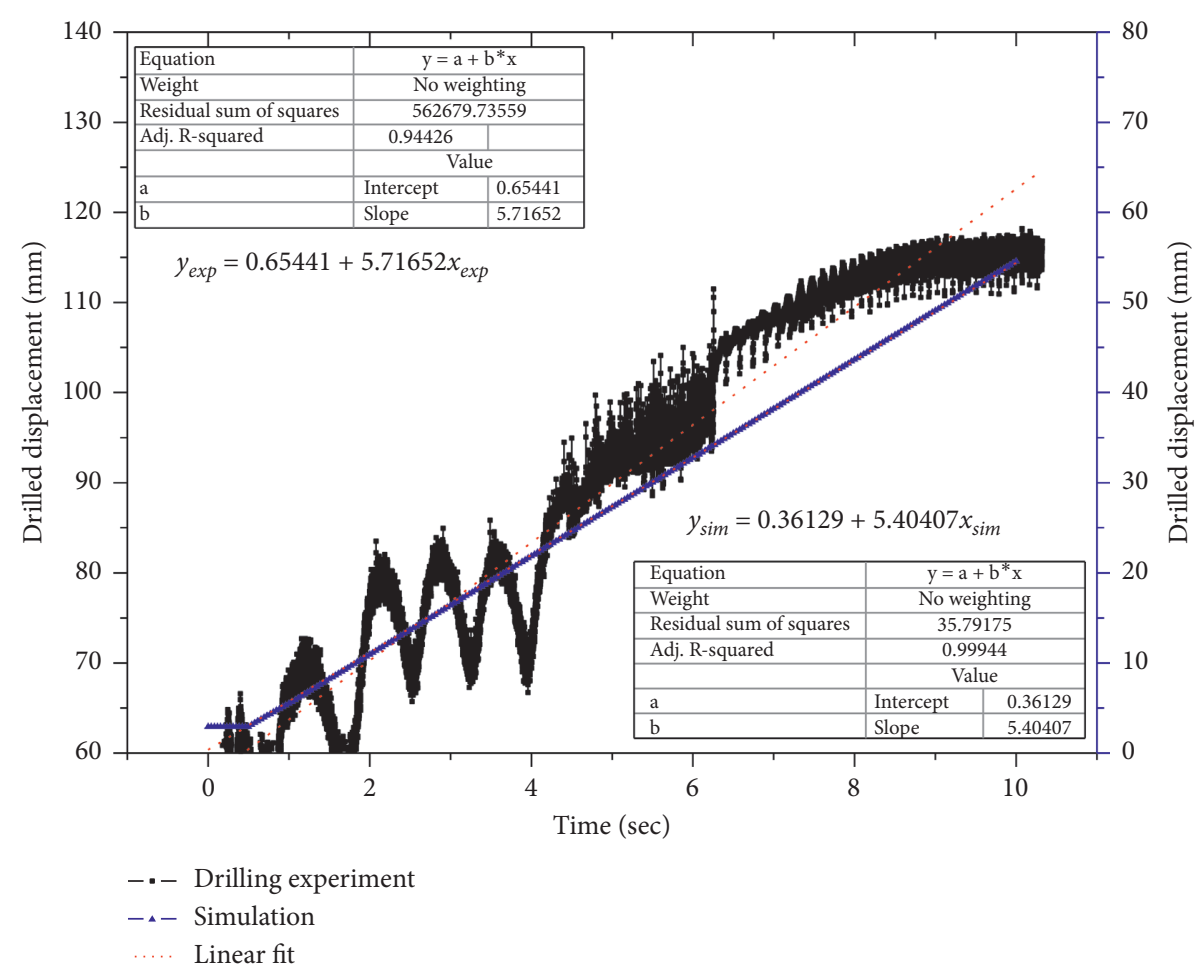

FIGURE 17: Comparison of predicted and measured drilling performances.

\section{Conclusions}

This study focused on predicting the drilling performance of a DTH hammer. A numerical analysis model of the DTH hammer was established and validated through comparison with the results of an in situ drilling test. The results of this work are summarized as follows:

(1) Simulations considering the effect of rock strength were used to quantify the energy efficiency according to the rock strength, impact energy required for crushing, and performance of the hammer.

(2) The developed model was applied to an actual DTH drilling system. The model predicted a drilling speed of $5.4 \mathrm{~mm} / \mathrm{s}$, and the measured speed was $5.7 \mathrm{~mm} / \mathrm{s}$. Similar results validated the proposed model. The model allows the performance of the drill bit and DTH hammer (i.e., the key components of the drilling system) to be predicted by considering the sensitivity of the major design factors and the effect of the rock mass.

(3) The validity of the proposed prediction model for the drilling performance was experimentally verified. The results of this study suggest that the performance of drilling equipment can be predicted by considering the sensitivity of major design factors for the drilling tool and the effect of the rock type.

Overall, these results suggest that considering the effects of key design factors can improve the performance of drilling equipment for soil or rock. The findings are expected to improve the performance of DTH hammers and serve as a basis for further research on the development of new rock drilling hammers.

Important factors that affect the performance of a percussive drilling system include the feed and rotation forces, the impact force of the percussive drilling tool, and the ground and rock properties. In this study, only the impact force and feed force acting on the button of the percussive drilling tool were considered. The operating conditions of the vehicle were not considered, such as the rotation force, dynamics of the drill rod (e.g., whirling motion) during deep drilling, drilling characteristics of various rocks, and overlapping drilling effect due to the use of multiple buttons. Because the prediction results of the proposed model slightly differed from the actual drilling performance, these conditions need to be considered. Furthermore, the drilling performance was quantified solely through the fractured rock volume and drilling rate. Further work should consider the effects of the DTH drilling system and various performance factors, such as the reaction and transfer force generated by the rock drilling device, to improve the proposed model. If studies are conducted to consider the various effects of drilling work, the drilling characteristics and borehole deviations can be analyzed to maximize the drilling and blasting efficiency. Further studies are required to identify the dynamic effects of drill rods and analyze the effect of borehole deviation and drill rod characteristics on the drilling performance. Tests need to be performed on various rocks to consider the actual working environment.

\section{Data Availability}

The simulation and experimental data used to support the findings of this study are included within the article. 


\section{Conflicts of Interest}

The authors declare that they have no conflicts of interest.

\section{Acknowledgments}

This work was supported by the Korea Agency for Infrastructure Technology Advancement (KAIA) grant funded by the Ministry of Land, Infrastructure and Transport (Grant 20SMIP-A157130-01).

\section{References}

[1] C. Song, J. Chung, J.-S. Cho, and Y.-J. Nam, “Optimal design parameters of a percussive drilling system for efficiency improvement," Advances in Materials Science and Engineering, vol. 2018, Article ID 2346598, 13 pages, 2018.

[2] C. Song, J. Chung, J.-H. Kim, and J.-Y. Oh, "Design optimization of a drifter using the Taguchi method for efficient percussion drilling," Journal of Mechanical Science and Technology, vol. 31, no. 4, pp. 1797-1803, 2017.

[3] J.-Y. Oh, G.-H. Lee, H.-S. Kang, and C.-S. Song, "Modeling and performance analysis of rock drill drifters for rock stiffness," International Journal of Precision Engineering and Manufacturing, vol. 13, no. 12, pp. 2187-2193, 2012.

[4] D.-Y. Shin and C.-H. Song, "Performance optimization of down-the-hole hammer using Taguchi method," Transactions of the Korean Society of Mechanical Engineers A, vol. 36, no. 1, pp. 109-116, 2012.

[5] B. Changgen, Q. Yegao, C. Zhiqiang, and L. Baolin, "Numerical simulation of impact on pneumatic DTH hammer percussive drilling," Journal of Earth Science, vol. 20, no. 5, pp. $868-878,2009$.

[6] L. E. Chiang and D. A. Elias, "A 3D FEM methodology for simulating the impact in rock-drilling hammers," International Journal of Rock Mechanics and Mining Sciences, vol. 45, no. 5, pp. 701-711, 2008.

[7] B. Lundberg and M. Okrouhlik, "Influence of 3D effects on the efficiency of percussive rock drilling," International Journal of Impact Engineering, vol. 25, no. 4, pp. 345-360, 2001.

[8] X. Li, G. Rupert, D. A. Summers, P. Santi, and D. Liu, "Analysis of impact hammer rebound to estimate rock drillability," Rock Mechanics and Rock Engineering, vol. 33, no. 1, pp. 1-13, 2000.

[9] X. Li, G. Rupert, D. A. Summers, P. Santi, and D. Liu, "Energy transmission of down-hole hammer tool and its conditionality," Transactions of Nonferrous Metals Society of China, vol. 10, no. 1, pp. 109-111, 2000.

[10] H. Kang, J.-Y. Park, J.-W. Cho, J.-S. Jang, K.-W. Kim, and J.-W. Lee, "Optimal button arrangement of a percussion drill bit and its operating condition for improving drilling efficiency," Proceedings of the Institution of Mechanical Engineers, Part C: Journal of Mechanical Engineering Science, vol. 232, no. 16, pp. 2887-2898, 2018.

[11] D.-J. Kim, J.-Y. Oh, J.-W. Cho, J. Kim, J. Chung, and C. Song, "Design study of impact performance of a DTH hammer using PQRSM and numerical simulation," Journal of $\mathrm{Me}$ chanical Science and Technology, vol. 33, no. 11, pp. 5589$5602,2019$.

[12] K.-B. Kwon, C.-H. Song, J.-Y. Park, J.-Y. Oh, J.-W. Lee, and J.-W. Cho, "Evaluation of drilling efficiency by percussion testing of a drill bit with new button arrangement," International Journal of Precision Engineering and Manufacturing, vol. 15, no. 6, pp. 1063-1068, 2014.
[13] K. Hashiba, K. Fukui, Y. Z. Liang, M. Koizumi, and T. Matsuda, "Force-penetration curves of a button bit generated during impact penetration into rock," International Journal of Impact Engineering, vol. 85, pp. 45-56, 2015.

[14] T. Saksala, D. Gomon, M. Hokka, and V.-T. Kuokkala, "Numerical and experimental study of percussive drilling with a triple-button bit on Kuru granite," International Journal of Impact Engineering, vol. 72, pp. 56-66, 2014.

[15] C. H. Song, K. B. Kwon, M. G. Cho, J. Y. Oh, D. Y. Shin, and J. W. Cho, "Development of lab-scale rock drill apparatus for testing performance of a drill bit," International Journal of Precision Engineering and Manufacturing, vol. 16, no. 7, pp. 1405-1414, 2015.

[16] M. Fourmeau, A. Kane, and M. Hokka, "Experimental and numerical study of drill bit drop tests on Kuru granite," Philosophical Transactions of the Royal Society A: Mathematical, Physical and Engineering Sciences, vol. 375, no. 2085, Article ID 20160176, 2017.

[17] J.-W. Cho, S. Jeon, S.-H. Yu, and S.-H. Chang, "Optimum spacing of TBM disc cutters: a numerical simulation using the three-dimensional dynamic fracturing method," Tunnelling and Underground Space Technology, vol. 25, no. 3, pp. 230244, 2010.

[18] K. B. Kwon, C. H. Song, J. Y. Park et al., "Rock fragmentation assessment of a drill bit by hopkinson bar percussion test," Journal of Korean Society For Rock Mechanics, vol. 23, no. 1, pp. 45-53, 2013.

[19] C. Song and Y. J. Nam, "Optimum Design of Impact Performance in a DTH Hammer by Numerical Simulation and Optimization Algorithm," in Proceedings of the Korea Society of Fluid Power \& Construction Equipments, 2018 Autumn Conference on Drive and Control, pp. 29-30, Gyeonggi-do, Republic of Korea, 2018.

[20] S. Kahraman, N. Bilgin, and C. Feridunoglu, "Dominant rock properties affecting the penetration rate of percussive drills," International Journal of Rock Mechanics and Mining Sciences, vol. 40, no. 5, pp. 711-723, 2003. 